\title{
PREDICTORES PSICOLÓGICOS Y SOCIODEMOGRÁFICOS DE LA ANSIEDAD ANTICIPATORIA ANTE LA PARTICIPACIÓN EN «SEGUNDAS PRUEBAS» DE DETECCIÓN DE CÁNCER DE MAMA
}

\author{
BONIFACIO SANDIN, PALOMA CHOROT, LOURDES LOSTAO ${ }^{1}$, \\ ROSA M. VALIENTE y MIGUEL A. SANTED
}

Facultad de Psicología, Universidad Nacional de Educación a Distancia

'Universidad Pública de Navarra

\section{(Aceptado en enero de 2001)}

\begin{abstract}
La presente investigación se ha centrado en estudiar la capacidad predictiva de las actitudes hipocondríacas, las variables sociodemográficas (edad, ingresos familiares, nivel educativo, estado civil, número de hijos, y área geográfica) y otras variables psicológicas (neuroticismo, locus de control hacia la salud, autoestima y apoyo social) sobre la ansiedad anticipatoria en mujeres (45-65 años de edad) que fueron citadas para repetir la prueba de detección de cáncer de mama. Análisis de regresión lineal y logística fueron empleados para estudiar los efectos de las variables independientes sobre la ansiedad anticipatoria asociada a la participación en la segunda prueba. En consonancia con nuestras hipótesis, la actitud hipocondríaca fue el mejor predictor de la ansiedad anticipatoria. Las escalas de actitud hacia la enfermedad pertenecientes a la dimensión de «miedo irreal a la enfermedad" (fobia a la enfermedad, tanatofobia) fueron mejores predictores que las escalas vinculadas a la dimensión de "convicción de tener una enfermedad" (creencias hipocondriacas, efectos de los síntomas, etc.). Otros predictores significativos fueron el neuroticismo y el locus de control hacia la salud centrado en la suerte. No encontramos efectos significativos para las variables de autoestima, apoyo social, y variables sociodemográficas. Finalmente, los resultados sugieren que el poder predictivo de la actitud hipocondríaca sobre la ansiedad anticipatoria es superior en el grupo de mujeres que participan en las pruebas complementarias que en el de mujeres que asisten a pruebas rutinarias de screening.
\end{abstract}

Palabras clave: Ansiedad anticipatoria, mamografía, cáncer de mama, screening, hipocondría, estrés, neuroticismo, locus de control hacia la salud, autoestima, apoyo social.

\section{Predictors of anticipatory anxiety in women attending a second stage breast cancer screening}

This study examined the predictive power of hypochondriacal attitudes, demographics (age, income, educational level, marital status, children, and rural vs. urban area) and other moderating variables (neuroticism, multidimensional health locus of control, self-esteem, and social support) on anticipatory anxiety in women (45-65 years old) following a "false positiven breast cancer screening result. Lineal and logistic regression analyses were used to study the effects of independent variables on anticipatory anxiety associated with attendance to the second stage screening. Consistent with our predictions, hypochondriacal attitude was the best predictor of anticipatory anxiety. Illness attitude scales that correspond to the "disease unrealistic fear" dimension (disease phobia, thanatophobia) were better predictors than those describing the "conviction of having a disease" (hypochondriacal beliefs, effects of symptoms, etc.). Other significant predictors were neuroticism and chance health locus of control. No significant effects were found to self-esteem, social support and demographics variables. Finally, findings suggest that predictive power of hypochondriacal attitude on anticipatory anxiety is better for women attending second stage screening than for those attending routine screening.

Key words: Anticipatory anxiety, mammography, breast cancer screening, hypochondriasis, stress, anxiety, neuroticism, health locus of control, self-esteem, social support.

Correspondencia: Bonifacio Sandín, Facultad de Psicologia, Universidad Nacional de Educación a Distancia (UNED), Ciudad Universitaria s/n, 28040 Madrid.

Agradecimientos: El presente estudio ha sido financiado con la ayuda a la investigación $n^{2} 2204$, resolu- ción 634/96, del Departamento de Salud del Gobierno de Navarra. Deseamos agradecer a la Dra. Nieves Ascunce y al Dr. Ángel Del Moral su inestimable ayuda y sugerencias para la realización de la presente investigación, y a la psicóloga Olga Fernández Soto su participación en la recogida de la información. 


\section{INTRODUCCIÓN}

El cáncer de mama constituye uno de los principales problemas de salud en la mayoría de los países occidentales. Como ha venido siendo indicado en diversas publicaciones científicas (Ascunce, 1991; Ascunce y Del Moral, 1993; Lostao, 1994; Luengo et al., 1996), esta enfermedad es una de las formas más frecuentes de cáncer en la mujer y una de las principales causas de muerte. Bien es cierto que, como han señalado algunos autores (p.ej., De Waard, 1993), España posee la tasa más baja de incidencia y mortalidad por cáncer de mama entre todos los países de la Unión Europea. No obstante, y aunque su prevalencia es menor que en otros países occidentales (los países mediterráneos ocupan, junto a España, las posiciones más bajas a este respecto; véase Lostao, 1994), el cáncer de mama constituye sin duda alguna un importante problema de salud pública.

Aunque existe cierta controversia al respecto, se asume que un fenómeno esencial para la curación del cáncer de mama es su detección precoz. El cáncer de mama suele poseer un peor pronóstico que otras formas de cáncer femenino (p.ej., el cáncer de cuello de útero) (Wardle y Pope, 1992). No obstante, se ha venido asumiendo que su detección precoz constituye un paso esencial para el tratamiento eficaz de esta modalidad de tumor al diagnosticarse en un estadio temprano de su desarrollo. En este sentido, se ha demostrado que la realización periódica de mamografías puede contribuir de forma significativa a la detección precoz del cáncer de mama. Esto ha permitido llevar a cabo tratamientos más tempranos del cáncer $\mathrm{y}$, con ello, una reducción significativa de la mortalidad debida a este tipo de enfermedad en mujeres por encima de los 50 años. Las cifras sobre las tasas de reducción de la mortalidad han cambia- do significativamente, tal vez debido a las variaciones debidas a la edad de las mujeres estudiadas. Aunque en términos generales la tasa de reducción de la mortalidad se ha situado en torno al $30 \%$ (Chamberlain y Palli, 1993), los datos suelen variar entre un 4 y un $40 \%$ (American Cancer Society, 1998).

Los programas de screening de cáncer generalmente suelen evaluarse en términos de «costes" y «beneficios». El principal beneficio de cualquier programa deriva de que, al permitir detectar el cáncer en estadios tempranos de desarrollo, el tratamiento puede ser más eficaz una vez que se halla en fases aún poco avanzadas. El descenso en el número de muertes debidas al cáncer de mama constituye una prueba en favor de este tipo de beneficios.

Los costes son evaluados habitualmente en términos de gastos de tiempo y dinero. Sin embargo, un tipo de coste que apenas ha sido investigado es el que concierne al coste psicológico, entendido éste generalmente en términos de estrés y distress (malestar y sufrimiento emocional) asociados al proceso del screening, sobre todo cuando se trata de personas sanas (Wardle y Pope, 1992). A pesar de que el impacto psicológico asociado al screening podría representar un fenómeno de gran relevancia, sólo recientemente ha comenzado a ser estudiado de forma más o menos sistemática.

Si bien aún son pocos los estudios que han analizado específicamente el impacto psicológico relacionado con la participación a las pruebas de mamografía, la evidencia es consistente con la idea de que, al menos para algunas personas, el hecho de participar en las pruebas rutinarias podría inducir niveles significativos de ansiedad y distress (Marteau, 1994). Se ha sugerido que el coste psicológico potencial puede oscilar entre el trauma que supone la identi- 
ficación de una enfermedad y el distress psicológico (ansiedad, preocupaciones, miedo, etc.) que pueden experimentar las mujeres sanas, sobre todo las que sospechan inicialmente la posibilidad de tener cáncer. De cualquier modo, el screening en sí mismo puede suponer un motivo de al menos un nivel moderado de preocupación, ansiedad o malestar en general (McCaul, Branstetter, Schroeder y Glasgow, 1996a; McCaul, Reid, Rathge, y Martinson, 1996b; Johnston, Brown, Gerard, O'Hanlon y Morton, 1998).

En principio, resulta lógico asumir que el hecho de recibir un resultado negativo del screening (i.e., diagnóstico de no poseer cáncer) debe ser tranquilizador para la mujer. Algunos autores, no obstante, han hipotetizado que esto no necesariamente ocurre de este modo. Para algunas personas, el mero hecho de participar en el screening puede ser un hecho suficiente como para albergar dudas sobre su propia salud, generándose cierto grado de incertidumbre (p.ej., Cioffi, 1991). Esta hipótesis estaría de acuerdo con el modelo propuesto por Marteau (1994) sobre la relación entre la incertidumbre y la emoción en el screening. Básicamente, el autor sugiere en este modelo que, en la población normal, la aplicación del screening facilita la génesis de incertidumbre y alta ansiedad, lo cual puede ocurrir a partir de estados de baja ansiedad y certidumbre sobre la propia salud.

Aunque escasas, se han llevado a cabo algunas investigaciones para estudiar el impacto psicológico asociado a la participación en pruebas rutinarias de screening de cáncer de mama. A partir de algunos estudios preliminares sobre esta cuestión parece deducirse que la participación en este tipo de pruebas no suele ser particularmente estresante. Así por ejemplo, Ellman et al., (1989) no encontraron cambios significativos en morbilidad psiquiátrica (puntuaciones en el
General Health Questionnaire de Goldberg) en un grupo de mujeres que participaron en un screening de cáncer de mama. De modo semejante, Dean, Roberts, French y Robinson (1986), basándose en entrevistas con mujeres que asistían a pruebas de screening de cáncer de mama, no hallaron diferencias en el número de casos psiquiátricos con respecto a un grupo de control procedente de la misma población de mujeres. Walker et al. (1994) evaluaron la ansiedad y la depresión relativas a la semana previa al screening de cáncer de mama, no encontrando incrementos de estas emociones durante tal periodo de tiempo.

Un problema diferente asociado a los programas de detección de cáncer de mama viene dado por la existencia de "pruebas complementarias», o segundas pruebas de screening (i.e., repetición de las pruebas mamográficas). Estas pruebas se llevan a cabo cuando los resultados de la mamografía rutinarias no son claros (p.ej., por baja calidad de la mamografía) o reflejan algún signo de patología (p.ej., por sospecha de riesgo de cáncer). El número de mujeres que suelen someterse a este tipo de pruebas suele ser elevado. Aunque las estimaciones han variado entre unos y otros autores, el porcentaje de mujeres que deben repetir las pruebas se sitúa en torno al 10\% (Clutton, Pakenham y Buckley, 1999; Wardle y Pope, 1992).

De acuerdo con el modelo de emocióncognición de Marteau (1994), podría afirmarse que la aplicación de segundas pruebas de screening, a mujeres de la población general, constituye una situación paradigmática de generación de incertidumbre $y$, consecuentemente, de elevada ansiedad anticipatoria. De hecho, como se deduce de este modelo, la incertidumbre sobre los resultados de una prueba puede asociarse con mayor grado de ansiedad y preocupación sobre la salud que el recibir un resultado clara- 
mente positivo o negativo. Puesto que las mujeres que deben superar una segunda prueba mamográfica (i.e., la prueba complementaria) están temporalmente en una situación de más o menos incertidumbre, los efectos de impacto psicológico (ansiedad, distress, etc.) podrían, en principio, ser elevados. Debido a la incertidumbre que suele inducir una situación de este tipo, es probable que las mujeres afectadas experimenten niveles elevados de afectación psicológica, especialmente de ansiedad anticipatoria. La necesidad de ser requerida para repetir las prueba puede generar en la mujer una situación de "falso positivo" (i.e., la mujer puede experimentar temporalmente la idea de padecer cáncer) (Skrabaneck, 1985). Como ha referido Warren (1988), «una mujer que recibe una carta para repetir la prueba experimenta temporalmente el diagnóstico de cáncer. Tres casos de repetición por cada caso de cáncer son aceptables, pero 10 o 20 no lo son".

Aunque se trata de un fenómeno de enorme relevancia desde el punto de vista de la salud comunitaria, son pocas las investigaciones que han abordado directa o indirectamente el estudio del impacto psicológico vinculado a la repetición de las pruebas en programas de detección de cáncer de mama. No es extraño, por tanto, que los resultados que se han referido sean escasamente concluyentes. Así por ejemplo, mientras que Ellman et al (1989) no encontraron diferencias significativas en morbilidad psiquiátrica entre mujeres que participaban en pruebas rutinarias y mujeres que asistian a pruebas complementarias, otros autores, como Cockburn, Staples, Hurley y De Luise (1994), Lerman, Trock, Jepson, Brody y Boyce (1991) y Sutton, Saidi, Bickler y Hunter (1995) constataron que las mujeres que participaban en las segundas pruebas sufrían mayor impacto psicológico que las que asistían a las pruebas rutinarias.
Aunque, en principio, la participación a pruebas complementarias parece que podrían generar un nivel importante de impacto emocional (ansiedad, preocupación, distress, morbilidad psiquiátrica, etc.), sobre todo debido a su posible identificación, aunque sólo temporalmente, con el concepto de "falso positivo» y, a su vez, debido al elevado nivel potencial de incertidumbre que conllevan, los escasos estudios que han abordado empíricamente este problema, bien de forma directa o indirecta, no aportan datos consistentes que permitan establecer conclusiones válidas y posibiliten establecer acciones prácticas dirigidas a mejorar el funcionamiento de los programas de screening y la calidad de vida de las mujeres afectadas.

Dada la necesidad de investigar de forma específica este problema, nuestro grupo ha llevado a cabo recientemente un proyecto de investigación longitudinal breve dirigido a estudiar la afectación psicológica asociada a la participación a segundas pruebas de screening de cáncer de mama. El proyecto se ha desarrollado vinculado al Programa de Detección de Cáncer de Mama (PDCM) del Gobierno de Navarra (Ascunce y Del Moral, 1993). Inicialmente constatamos algunos resultados interesantes, entre los cuales merece la pena resaltar los siguientes (Sandín, Chorot, Valiente, Lostao y Santed, 2001a): (a) Parece producirse un grado significativo de afectación psicológica, fundamentalmente en lo que concierne a la ansiedad anticipatoria que experimentan las mujeres durante los días que preceden a la realización de la prueba complementaria (i.e., en el intervalo de tiempo que transcurre entre la citación y la participación en dicha prueba). (b) Las mujeres que acuden a pruebas complementarias sufren mayores niveles de ansiedad anticipatoria (evaluada tanto a través de autorregistro como mediante cuestionario de autoinforme) que las mujeres que acuden 
únicamente a las pruebas rutinarias de screening. (c) Esta afectación asociada a las pruebas complementarias parece extinguirse transcurrido poco tiempo después de la realización de la prueba y haber recibido la información tranquilizadora (información proporcionada por el médico sobre la inexistencia de cáncer).

Posteriormente, hemos obtenido resultados semejantes para otras variables de afectación psicológica (impacto afectivocognitivo), tales como la preocupación por la posibilidad de tener cáncer, el miedo a tener cáncer, y la creencia de tener cáncer (vulnerabilidad percibida) (Sandín, Chorot, Valiente, Lostao y Santed, 2001b). De acuerdo con los resultados hallados en este segundo estudio, las mujeres citadas para repetir la mamografía sufrían niveles superiores de impacto afectivo-cognitivo que las mujeres que participaban únicamente en las pruebas rutinarias de screening. Las diferencias se obtuvieron para las tres medidas, i.e., preocupación, miedo y vulnerabilidad percibida. Otro hallazgo importante de este estudio fue la constatación de que tal afectación sólo se producía durante el periodo previo a la mamografía, esto es, desde que la mujer era citada para repetir la mamografía hasta la realización de la misma (momento en que era informada por el médico sobre la inexistencia de resultados significativos). El distress afectivo-cognitivo no parecía persistir dos días después de la notificación de los resultados ni durante el periodo de seguimiento (dos meses después de la notificación).

En cambio, hemos constatado que la afectación psicológica es muy baja o inexistente para las variables que poseen una significación clínica más marcada, tales como la depresión, la sintomatología somática, la morbilidad psicopatológica o las actitudes hipocondríacas (Sandín et al., 2000, 2001a, 2001b). En relación con este tipo de variables, no sólo no hemos encontrado diferencias significativas entre las mujeres que acuden a las pruebas complementarias y las que lo hacen a las pruebas rutinarias, sino que el grado de afectación resulta ser muy bajo o irrelevante.

Por tanto, nuestros resultados parecen poner de relieve la existencia de cierto impacto emocional y cognitivo asociado a la participación en pruebas complementarias de screening de cáncer de mama. Dicha afectación es moderada y posee corta duración, ya que se limita al periodo de tiempo que transcurre entre la nueva citación y la repetición de la prueba e información sobre la inexistencia de cáncer. Merece la pena señalar que un porcentaje importante de mujeres que acuden a realizar dichas pruebas presenta niveles elevados de ansiedad, ánimo depresivo, preocupación por el cáncer, miedo a tener cáncer, y creencia de tener cáncer (vulnerabilidad percibida); por ejemplo, cerca del $50 \%$ de las mujeres refieren niveles entre moderados y altos de ansiedad durante los dos días que preceden a la realización de la prueba (Sandín et al., 2001a). Por tanto, aunque no parece confirmarse la hipótesis señalada por algunos autores (Schmith, 1990; Skrabaneck, 1985) de que el screening de cáncer de mama, y en particular el screening asociado a las pruebas complementarias, al poder generar la expectativa de "falso positivo», podría generar perturbaciones psicológicas serias, sí parece cierto que en algún grado puede reducir la calidad de vida de las mujeres, al perturbar ciertas variables relacionadas con el bienestar emocional.

Basándonos en la existencia de dicha afectación psicológica y en la consideración de que las segundas pruebas de screening pueden constituir una fuente importante de estrés, en nuestro último estudio analizamos la capacidad que poseen algunas variables moduladoras del estrés para predecir el impacto psico- 
lógico producido por las pruebas complementarias (Sandín, Valiente, Chorot, Lostao y Santed, 2001c). Existen diversos factores personales y sociales sobre los cuales existe evidencia de su papel modulador en las relaciones entre el estrés y la salud, entre los cuales se incluyen el neuroticismo, la autoestima, las actitudes hipocondríacas, el locus de control hacia la salud y el apoyo social (Belloch y Martínez, 1999; Clutton et al., 1999; Kellner, 1986; Sandín, 1995; Sandín, Valiente y Chorot, 1999; Taylor, 1995). Cabría pensar, por consiguiente, en la capacidad de estas variables para predecir el nivel de afectación emocional asociado a la participación en las pruebas complementarias. Dicho en otros términos, se trataba de estudiar qué tipo de mujeres resultaban ser más vulnerables para experimentar la afectación cognitivo-emocional (ansiedad, ánimo depresivo, preocupación, miedo, y vulnerabilidad percibida).

A pesar de utilizar diversos tipos de variables moduladoras (i.e., neuroticismo, autoestima, actitudes hipocondríacas, locus de control hacia la salud, y apoyo social), mediante diferentes análisis de regresión múltiple encontramos que las únicas variables moduladoras que resultaron ser realmente importantes para predecir la afectación afectivo-cognitiva eran las actitudes hipocondríacas y el neuroticismo. Más aun, si bien parece resultar relevante el empleo del índice global de hipocondría (i.e., IAS global), algunas dimensiones o subescalas hipocondríacas parecían resultar más relevantes que otras. Una de las principales dimensiones hipocondríacas predictora de la afectación emocional resultó ser la fobia a la enfermedad. Otras dimensiones hipocondríacas particularmente relevantes en este sentido fueron la tanatofobia (i.e., fobia a la muerte) y la preocupación por la enfermedad.

Merece la pena resaltar que, entre las diferentes dimensiones hipocondríacas, las que mejor parecen predecir el impacto emocional ante las pruebas complementarias eran las implicadas en el factor de hipocondría que hemos denominado como dimensión de «fobia a la enfermedad», en contraste con la dimensión de "convicción de enfermedad" (Belloch y Martínez, 1999; Chorot, Sandín, Valiente, Santed y Romero, 1997; Martínez y Belloch, 1993). En este reciente estudio llevado a cabo por nuestro grupo de la UNED, basándonos en el cuestionario IAS, constatamos dos dimensiones factoriales de la hipocondría, la dimensión de «fobia a la enfermedad" y la dimensión de «convicción de enfermedad». La dimensión de fobia a la enfermedad estaba configurada fundamentalmente por las subescalas de fobia a la enfermedad, tanatofobia y preocupación por la enfermedad. A su vez, el factor de convicción de enfermedad estaba constituido sobre todo por las subescalas creencias hipocondríacas, efecto de los síntomas y experiencia del tratamiento. Las subescalas relativas a la preocupación por el dolor y a las preocupaciones corporales saturaban en ambos factores (Chorot et al., 1997).

En suma, del análisis de nuestros resultados se desprende que las mujeres que participan en pruebas complementarias de screening de cáncer de mama sufren mayor nivel de afectación afectivo-cognitiva cuando son más hipocondríacas y más neuróticas. Entre las subescalas de las actitudes hipocondríacas, la actitud de fobia o miedo a la enfermedad parecía resultar más relevante que otras dimensiones. Las actitudes hacia la enfermedad (o actitudes hipocondríacas), y secundariamente el neuroticismo, resultaron ser las principales variables personales predictoras del impacto psicológico asociado a la participación en las pruebas complementarias. Curiosamente, las actitudes hipocondríacas también han resultado ser particularmente relevantes para predecir 
la participación de las mujeres en las pruebas rutinarias del PDCM de Navarra (Lostao, 1994). Según se desprende del estudio de esta autora, las mujeres que declinaban participar en las pruebas rutinarias de detección de cáncer de mama presentaban puntuaciones significativamente más elevadas en actitudes hipocondríacas que las mujeres que participaban habitualmente. Estas variables eran altamente predictoras de la no participación en las pruebas de screening.

Mediante el presente estudio, el cual es una continuación del llevado a cabo por Sandín et al. (2001c), pretendemos examinar la capacidad predictiva de las mencionadas variables psicológicas específicamente sobre la ansiedad anticipatoria. Ésta es una variable combinada que hemos calculado sumando los valores correspondientes a tres variables de afectación psicológica, esto es, el estado de ansiedad (STAI-E), la ansiedad/nerviosismo, la preocupación por el cáncer, y el miedo a tener cáncer (véase la sección del método). Estas cuatro variables, aparte de relacionarse teóricamente con el constructo de "ansiedad anticipatoria», también presentan entre sí elevadas correlaciones (p.ej., en el grupo de mujeres que participaban en pruebas complementarias, las correlaciones entre dichas variables oscilaban entre 0,64 y 0,97). Así mismo, a partir de esta variable de ansiedad anticipatoria podría calcularse, no sólo la capacidad predictiva de las variables moduladoras indicadas sobre la experiencia de distress o ansiedad anticipatoria, sino también los niveles de «riesgo» de tales variables en relación con la posibilidad de sufrir la experiencia emocional negativa. Por otra parte, en el presente estudio examinamos la posible relación que poseen las variables sociodemográficas con la ansiedad anticipatoria.

En nuestro anterior estudio observamos que las diferentes subescalas de acti- tud hacia la enfermedad (i.e., subescalas del IAS; véase la sección del método) no predecían de forma idéntica las distintas variables de afectación psicológica (Sandín et al., 2001c). Por tanto, en una primera hipótesis deseábamos conocer si las subescalas hipocondríacas más vinculadas con la dimensión de "miedo a la enfermedad" (miedo irreal a tener una enfermedad) eran mejores predictores de la ansiedad anticipatoria y/o mayores factores de riesgo que las subescalas hipocondríacas pertenecientes a la dimensión de "convicción de enfermedad" (convicción de tener una enfermedad).

Nuestra segunda hipótesis implicaba la consideración de las variables sociodemográficas como posibles factores predictores. Aunque no se han publicado estudios que hayan analizado la relación entre las variables sociodemográficas y la afectación psicológica ante las segundas pruebas de screening, existe evidencia de que algunas de estas variables (p.ej., estado civil, nivel educativo, nivel económico, nivel asociativo, zona geográfica) parecen relacionarse con el nivel de participación en las pruebas rutinarias de screening (Lostao, 1994; Lostao, Chorot, Sandín y Lacabe, 1996; Luengo et al., 1996), así como también con los efectos emocionales del estrés (Sandín, 1999). Basándonos en este tipo de evidencia, hipotetizamos que las variables ingresos familiares, nivel de estudios, edad, y número de hijos deberían relacionarse de forma inversa con la ansiedad anticipatoria. En cuanto a la variable estado civil, estar casada o en pareja debería relacionarse de forma inversa con la ansiedad anticipatoria, mientras que estar soltera, viuda, o separada/divorciada deberían asociarse positivamente con la ansiedad anticipatoria. En relación con la variable zona (rural vs. urbana), hipotetizamos que la pertenencia a áreas rurales debería potenciar la experiencia de ansiedad anticipatoria. 
Finalmente, nuestra tercera hipótesis se estableció a partir de los resultados de nuestro estudio previo (Sandín et al., 2001c), según los cuales la puntuación global en actitud hacia la enfermedad (i.e., puntuación total en el cuestionario IAS) era el principal y mejor predictor psicológico de las diferentes variables de afectación psicológica en las mujeres que acudían a realizar las pruebas complementarias. Puesto que la actitud hacia la enfermedad (i.e., actitudes hipocondríacas) es una variable de diferencias individuales que predispone específicamente hacia la manifestación de reacciones de distress ante estímulos o situaciones de amenaza para la salud (Belloch y Martínez, 1999; Chorot y Martínez, 1995; Kellner, 1986), hipotetizamos que dicha variable debería predecir la ansiedad anticipatoria mejor en las mujeres que acuden a las pruebas complementarias que las que participan en las pruebas rutinarias de screening de cáncer de mama. Para probar esta tercera hipótesis se incluyo en el estudio un grupo de este segundo tipo de mujeres, en adición al grupo de mujeres que participaban en las pruebas complementarias.

\section{MÉTODO}

\section{Participantes}

Se empleó una muestra de mujeres con un rango de edad entre 45 y 65 años, y participantes en el PDCM de Navarra. Se establecieron dos grupos. Un primer grupo $(N=600)$ correspondía a mujeres que asistieron consecutivamente al PDCM para realizar pruebas complementarias (i.e., segundas pruebas) [grupo de pruebas complementarias (PC)]. Estas mujeres fueron llamadas para repetir la prueba debido a que los resultados de la prueba rutinaria habían sido insatisfactorios, inconclusos o anormales. Un segundo grupo $(N=600) \mathrm{con}$ cernía a mujeres que participaron en la prueba rutinaria de screening, pero que no fueron citadas para repetir la prueba [grupo de pruebas rutinarias $(P R)$ ]. Las 600 mujeres de este segundo grupo fueron asignadas al azar a partir de una muestra de 1600 mujeres obtenidas consecutivamente al acudir a las pruebas rutinarias de screening. Tres mujeres del grupo PC y dos del grupo PR fueron diagnosticadas de cáncer de mama y, en consecuencia, fueron eliminadas del estudio. Por tanto, el número definitivo de mujeres fue de 597 (grupo PC) y 598 (grupo PR).

\section{Pruebas psicológicas}

(a) Variables sociodemográficas. Las variables sociodemográficas se evaluaron a través de una ficha personal de entrevista. Fueron categorizadas del siguiente modo (entre paréntesis se indican las categorías): zona geográfica (rural, urbana), edad (45-50, 51-55, 56-60, y 61-65 años), número de hijos (ninguno, uno, dos, tres, cuatro, y más de cuatro), ingresos familiares mensuales (menos de 100.000 , entre 100.000 y 250.000 , entre 250.000 y 400.000 , y más de 400.000 Ptas.), nivel de estudios (menor de primarios, primarios, formación profesional o bachiller elemental, bachiller superior, y universitarios), y estado civil (casada o en pareja, soltera, viuda, y separada o divorciada).

(b) Variables de autorregistro. Mediante una prueba de autorregistro se evaluó (1) la ansiedad/nerviosismo ( « ¿Hasta qué punto se ha sentido ansiosa o nerviosa?»), (2) la preocupación por el cáncer ( ¿Hasta qué punto le ha preocupado tener cáncer?», y (3) el miedo a tener cáncer ("¿Hasta qué punto ha tenido miedo de tener cáncer?»). Estas variables se 
puntuaron según una escala calibrada entre «Nada» (1) y «Muchísimo» (10), y se referían a los dos días previos a la prueba mamográfica. Se obtuvo una variable combinada de ansiedad anticipatoria sumando los valores de estas tres variables de autorregistro además del estado de ansiedad (STAI-E).

(c) Cuestionario de Ansiedad EstadoRasgo STAI. Se utilizó la versión española adaptada por TEA (Spielberger, Gorsuch y Lushene, 1982). Aplicamos la Parte 1 del STAI, la cual evalúa específicamente el estado de ansiedad. Esta parte (STAI-E) consta de 20 items, los cuales fueron contestados por los participantes indicando cómo se sentían durante los dos días previos según una escala de 5 niveles que variaba entre "Nada" (0) y "Muchísimo» (4).

(d) Escalas de Actitud hacia la Enfermedad (Illness Attitude Scales, IAS; Kellner, 1986). Consta de 29 items, de los cuales 5 corresponden a la subescala de experiencia de tratamiento (dimensión no evaluada en este estudio). Los restantes 24 items del IAS permiten evaluar las siguientes subescalas ( 3 items por escala):

1. Preocupación por la enfermedad (p.ej., « ¿Le asusta la idea de una enfermedad grave?»).

2. Preocupación por el dolor (p.ej., "¿Cree que tiene alguna enfermedad grave cuando un dolor o molestia no le desaparece después de una semana?»).

3. Hábitos de salud (p.ej., "¿Suele examinar su cuerpo para ver si tiene algo mal?»).

4. Creencias hipocondríacas (p.ej., «¿Cree que tiene alguna enfermedad física pero que los médicos no se la han diagnosticado correctamente?»).

5. Tanatofobia (p.ej., "¿Le asusta pensar en la muerte?»).

6. Fobia a la enfermedad (p.ej., “¿Le da miedo el que Ud. pueda tener una enfermedad del corazón?»).
7. Preocupaciones corporales (p.ej., «Cuando percibe alguna sensación en su cuerpo, ¿le resulta difícil pensar en otra cosa?»).

8. Efecto de los síntomas (p.ej., "¿Le impiden sus síntomas corporales que Ud. disfrute, que lo pase bien?»).

Cada uno de los items se puntúan según una escala Likert con rango entre 0 ("Nunca») y 4 («Casi siempre»). Así, el rango de valores para cada una de las subescalas es de 0-12, y el rango para la puntuación global en el IAS es de 0-96. Aplicamos una versión española de Chorot y Sandín (1989), la cual posee propiedades aceptables sobre fiabilidad y validez (Chorot et al., 1997; Lostao, 1994). En la Tabla 1 indicamos los valores de los coeficientes alfa obtenidos en el presente estudio.

(e) Cuestionario de Personalidad EPQR$A$ (Revised Eysenck Personality Questionnaire-Abbreviated). (Francis, Brown y Philipchalk, 1992; versión española de Sandín et al., 1999). Mediante esta forma abreviada del EPQ pueden evaluarse en poco tiempo las 3 dimensionas básicas de personalidad (neuroticismo, extraversión y psicoticismo), si bien en el presente estudio sólo se ha empleado la dimensión de neuroticismo. El EPQR-A consta de 24 items, 6 para cada dimensión. La fiabilidad, validez y estructura factorial de la versión española del EPQR-A han sido documentadas apropiadamente (Valiente, Sandín, Chorot y Santed, 1996, 1997).

(f) Escala de Autoestima SES [Selfesteem Scale; Rosenberg, 1965; versión española de Chorot y Navas, 1995 (véase Sandín et al., 1999)]. Es la escala clásica y más utilizada para evaluar el nivel de autoestima. Además se trata de un instrumento breve y fácil de aplicar. Consta de 10 elementos que se responden según una escala de cuatro 
puntos, variando entre 1 ( $«$ Muy en desacuerdo») y 4 («Muy de acuerdo»). El coeficiente alfa de fiabilidad de la SES obtenido a partir de la presente muestra de mujeres ha sido elevado $(\mathrm{a}=0,95)$.

(g) Escala Multidimensional de Locus de Control hacia la Salud (MHLC, Multidimensional Health Locus of Control Scales, Wallston, Wallston y DeVellis, 1978). Se aplicó la versión española de Chorot y Navas, 1995 (véase Sandín et al., 1999). Este cuestionario, en lugar de evaluar el tradicional locus de control (i.e., externo vs. interno), evalúa el «locus de control hacia la salud» según las tres dimensiones siguientes:

1. Interno (p.ej., "Cuando me encuentro enferma yo tengo la culpa»).

2. La suerte (p.ej., «Mi buena salud es en gran parte cuestión de buena suerten).

3. Otras personas (El poder/influencia de los otros) (p.ej., «La mejor forma de evitar una enfermedad es tener un contacto regular con el médico»).

Puesto que mediante la MHLC puede evaluarse el locus de control asociado a la salud, en investigaciones relacionadas con esta variable la MHLC es preferible a las más conocidas escalas sobre locus de control en general. El cuestionario consta de 18 elementos, correspondiendo 6 a cada una de las tres dimensiones. Se contestan según una escala de seis puntos, variando entre 1 ("Completamente en desacuerdo») y 6 ("Completamente de acuerdo»). Sandín, Valiente, Chorot y Lostao (1998) han referido datos positivos sobre su fiabilidad y validez. En la Tabla 1 indicamos los coeficientes alfa de fiabilidad obtenidos en el presente estudio.

(h) Escala de Apoyo Social SSB (The Social Support Behaviors (SSB) Scale; Vaux, Riedel y Stewart, 1987). Versión española de Sandín y Chorot, 1990 (Sandín et al., 1999). Evalúa el apoyo social percibido aportado por familiares y amigos, respectivamente. Datos sobre la fiabilidad y validez del cuestionario han sido presentados por nuestro grupo (Jiménez, Sandín, Chorot y Santed, 1994). Los datos sobre fiabilidad obtenidos en el presente estudio se refieren en la Tabla 1.

\section{Procedimiento}

La evaluación psicológica se llevó a cabo a través de entrevistas individualizadas «cara a cara». Todas las pruebas indicadas se aplicaron durante la fase de premamografía (otras fases posteriores no son consideradas para el presente estudio). Las mujeres fueron entrevistadas mediante un protocolo en el que se incluían las mencionadas pruebas psicológicas.

Todas las participantes eran informadas de los resultados de la mamografía. Las mujeres citadas para las pruebas complementarias recibían la información justo después de haber concluido la entrevista y la realización de las pruebas médicas. De acuerdo con el funcionamiento del PDCM de Navarra, las mujeres que acudían a realizar las pruebas rutinarias recibían la notificación por vía postal, la cual solía llegar en un plazo aproximado no superior de 15 días, a no ser que la mamografía presentase resultados equívocos o anormales (sospecha de patología), en cuyo caso eran citadas por teléfono para repetir las pruebas en el plazo de dos días.

\section{RESULTADOS}

Para probar las dos primeras hipótesis aplicamos inicialmente un modelo de 
análisis de regresión múltiple, en el que se incluyeron, como variables independientes (predictores), tanto las variables sociodemográficas (zona, estado civil, edad, ingresos familiares, y nivel de estudios, y número de hijos) como las variables psicológicas moduladoras (actitudes hacia la enfermedad, neuroticismo, autoestima, locus de control hacia la salud, y apoyo social), siendo la ansiedad anticipatoria la variable dependiente. Este modelo se aplicó empleando el método de pasos sucesivos. Posteriormente se calcularon análisis de regresión logística con objeto de estudiar las tasas de posibilidad de riesgo (odds ratio), debidas a las variables independientes (psicológicas y sociodemográficas), para experimentar la ansiedad anticipatoria (i.e., estar o no ansiosa). Para ello, esta variable fue dicotomizada en valores de cero (no-ansiedad) y uno (ansiedad), según que la puntuación fuese inferior o igual/superior a la media más una desviación típica. En un primer modelo de análisis de regresión logística, se incluyeron como variables independientes todas variables sociodemográficas de forma separada (análisis de regresión logística simple), con objeto de depurar las variables relevantes y observar la relevancia por separado de cada una de estas variables. En un segundo modelo (análisis de regresión logística múltiple) se incluyeron las variables sociodemográficas relevantes junto a todas las variables psicológicas. Previamente a los análisis de regresión lineal y logística se calcularon análisis de correlación y de varianza para ayudar a clarificar el sentido de la relación entre las variables predictoras y la variable criterio. Finalmente, para probar la tercera hipótesis se aplicaron análisis de regresión lineal independientes para el grupo PC y para el grupo PR, siendo en ambos casos la puntuación total en el IAS la variable independiente y el nivel de ansiedad anticipatoria la variable dependiente.

En la Tabla 1 se indican los coeficientes de fiabilidad alfa correspondientes a todas las variables psicológicas utilizadas, así como también las correlaciones de Pearson entre estas variables y la ansiedad anticipatoria. En lo que concierne a este último aspecto, puede observarse que la mayor parte de las correlaciones son estadísticamente significativas, siendo superiores las correlaciones con las variables de actitud hacia la enfermedad y neuroticismo que las concernientes al resto de variables moduladoras. De hecho, las correlaciones entre las variables de apoyo social y ansiedad anticipatoria son prácticamente irrelevantes. El signo de la correlación es congruente con lo que cabría esperarse para todas y cada una de las variables. Aparte de las elevadas correlaciones con las variables de actitud hacia la enfermedad y neuroticismo, merece la pena resaltar la correlación negativa con la autoestima y la correlación positiva con el locus de control «la suerte» (MHLC-Suerte). Por tanto, del presente análisis parece derivarse que las personas más hipocondríacas, más neuróticas, más conscientes de que la salud depende del azar, y con menor autoestima, son las que tienden a experimentar mayores niveles de ansiedad anticipatoria ante las segundas pruebas del screening.

En la Tabla 2 exponemos las correlaciones de Spearman entre la ansiedad anticipatoria y las variables sociodemográficas (variables categóricas) (se ha excluido la variable de estado civil debido a que ésta posee categorías independientes y carecerían de interpretación correlacional). Como se indica en la tabla, la ansiedad anticipatoria sólo ha correlacionado significativamente con las variables zona geográfica y nivel de estudios. La correlación negativa entre 
Tabla 1. Correlaciones de Pearson entre las variables psicológicas moduladoras y la ansiedad anticipatoria en el grupo de mujeres $(\mathrm{N}=597)$ que asistieron a pruebas complementarias de detección de cáncer de mama. También se indican los coeficientes alfa de Cronbach para las escalas y subescalas psicológicas

\begin{tabular}{|c|c|c|}
\hline Variable moduladora & $r$ de Pearson & Coeficiente alfa \\
\hline $\begin{array}{l}\text { IAS total (24) } \\
\text { IAS-Preocupación por la enfermedad (3) } \\
\text { IAS-Preocupación por el dolor (3) } \\
\text { IAS-Hábitos de salud (3) } \\
\text { IAS-Creencias hipocondríacas (3) } \\
\text { IAS-Tanatofobia (3) } \\
\text { IAS-Fobia a la enfermedad (3) } \\
\text { IAS-Preocupaciones corporales (3) } \\
\text { IAS-Efectos síntomas (3) }\end{array}$ & $\begin{array}{l}0,48 * * \\
0,39 * * \\
0,34 * * \\
0,16 * * \\
0,21 * * \\
0,38 * * \\
0,43 * * \\
0,39 * * \\
0,27 * *\end{array}$ & $\begin{array}{l}0,90 \\
0,86 \\
0,67 \\
0,25 \\
0,65 \\
0,82 \\
0,86 \\
0,87 \\
0,82\end{array}$ \\
\hline $\begin{array}{l}\text { EPQR-A-Neuroticismo (6) } \\
\text { MHLC-Interno (6) } \\
\text { MHLC-Suerte (6) } \\
\text { MHLC-Otros (6) } \\
\text { SES-Autoestima (10) } \\
\text { SSB-Amigos (45) } \\
\text { SSB-Familia (45) }\end{array}$ & $\begin{array}{l}0,31 \text { ** } \\
-0,07 \\
0,23 \text { ** } \\
0,12 \text { ** } \\
-0,17 \text { * } \\
-0,03 \\
-0,09 \text { * }\end{array}$ & $\begin{array}{l}0,60 \\
0,83 \\
0,57 \\
0,73 \\
0,95 \\
0,99 \\
0,99\end{array}$ \\
\hline
\end{tabular}

Nota: IAS = Escalas de Actitud hacia la Enfermedad, EPQR-A = Cuestionario de Personalidad de Eysenck Revisado-Abreviado, MHLC = Locus de Control Multidimensional hacia la Salud, SES = Escala de autoestima, $S S B$ = Escala de Apoyo Social. Entre paréntesis se indica el número de items de cada escala. ${ }^{\star \star} p<0,01$

zona geográfica y ansiedad indica que las mujeres procedentes de zonas rurales tienden a experimentar más ansiedad que las procedentes de zonas urbanas. Así mismo, las mujeres con niveles de estudios más bajos suelen experimentar mayor nivel de ansiedad anticipatoria que las mujeres de mayor estatus educativo. Aunque, como cabría suponer, el nivel de ingresos familiares se relaciona negativamente con el nivel de ansiedad, la correlación no alcanzó en nivel de significación estadística.
Puesto que las categorías de la variable sociodemográfica estado civil no permitían una interpretación correlacional, se efectuó un análisis de varianza (ANOVA) unifactorial de cuatro niveles (casada/en pareja, soltera, viuda y separada/divorciada), siendo la ansiedad anticipatoria la variable dependiente. El ANOVA resultó ser estadísticamente significativo $[F(3,593)=4,76, p<0,01]$. Los valores medios (entre paréntesis DT) en ansiedad anticipatoria para las cuatro categorías fueron como sigue: casada/en pareja =

Tabla 2. Correlaciones de Spearman entre las variables categóricas sociodemográficas y la ansiedad anticipatoria en el grupo de mujeres $(\mathrm{N}=597)$ que asistieron a pruebas complementarias de detección de cáncer de mama

\begin{tabular}{lccccc}
\hline & $\begin{array}{c}\text { Ansiedad } \\
\text { anticipatoria }\end{array}$ & $\begin{array}{c}\text { Zona } \\
\text { geográfica }\end{array}$ & Edad & $\begin{array}{c}\text { Ingresos } \\
\text { familiares }\end{array}$ & $\begin{array}{c}\text { Nivel } \\
\text { de estudios }\end{array}$ \\
\hline Zona geográfica & $-0,12 * *$ & & & & \\
Edad & 0,03 & $-0,37 * *$ & & & \\
Ingresos familiares & $-0,07$ & $0,14 * *$ & $-0,36 * *$ & & \\
Nivel de estudios & $-0,13 *$ & $0,18 * *$ & $-0,21 * *$ & $0,42 * \star$ & \\
Número de hijos & $-0,07$ & $-0,03$ & $0,18 * *$ & $-0,01$ & $-0,27 * *$ \\
\hline
\end{tabular}

${ }^{a}$ Se cuantificó como 1 para la procedencia urbana y como 0 para la procedencia rural. ${ }^{*} p<0,05 .{ }^{* *} p<0,01$ 
$46,9(13,3)$, soltera $=42,1(13,5)$, viuda $=$ $49,5(14,8)$, y separada/divorciada $=41,3$ $(11,1)$. Tal y como puede observarse, los valores más altos en ansiedad parecen corresponder a la condición de estar viuda, y los más bajos a estar soltera y separada/divorciada. La aplicación posterior de pruebas post hoc de Bonferroni reveló la existencia de diferencias significativas $(p<0,05)$ entre las categorías de estar viuda y estar soltera.

Los resultados relativos al análisis de regresión múltiple por pasos sucesivos están indicados en la Tabla 3. En la tabla se incluyen únicamente las variables independientes (predictores) que resultaron ser estadísticamente significativas de acuerdo con el método de pasos sucesivos (criterio: probabilidad de $F$ para entrar $\leq 0,05$, para salir $\geq$ de 0,10 ), las cuales explicaban el $27 \%$ de la varianza total. En el modelo se incluyeron inicialmente todas las variables psicológicas y sociodemográficas como variables independientes (no se incluyó la puntuación total del IAS, aunque sí las relativas a sus diferentes subescalas). Como se indica en la tabla, tres de las variables significativas corresponden a subescalas del cuestionario IAS (fobia a la enfermedad, preocupaciones corporales, y tanatofobia), una al neuroticismo, y una última al locus de control «la suerte». Por otra parte, se observa que la mayor parte de la varianza explicada corresponde a la variable fobia a la enfermedad $y$, en segundo lugar, a las preocupaciones corporales. Las tres restantes variables añaden cada una sólo una pequeña proporción de varianza explicada. Nótese, por otra parte, que otras variables como la autoestima, el locus de control interno o el apoyo social no proporcionan ningún valor predictivo que sea estadísticamente significativo. Tampoco resulta significativo el efecto asociado a las variables sociodemográficas; ninguna de estas variables predice el grado de ansiedad anticipatoria cuando se incluyen en la ecuación discriminante junto a las variables psicológicas.

Los datos que resumimos en la Tabla 4 corresponden a seis análisis de regresión logística simple. Cada análisis incluye como variable independiente una de las variables sociodemográficas categóricas, y como variable dependiente el estar (1) o no (0) ansiosa durante el periodo que precede a la prueba complementaria. Sólo dos de estas seis variables (zona geográfica y estado civil) resultaron ser estadísticamente significativas ( $p<0,05)$, y una (número de hijos) se acercó al nivel de significación estadística. No obstante, como puede observarse en la tabla, la proporción de varianza explicada por estas variables es demasiado baja. En lo que respecta a los $O R$, la procedencia

Tabla 3. Predicción del nivel de ansiedad anticipatoria (variable dependiente) asociada a las pruebas complementarias a partir inicialmente de todas las variables moduladoras psicológicas

y sociodemográficas (predictores) [análisis de regresión múltiple por el método de pasos sucesivos]

\begin{tabular}{lcccccc}
\hline Predictor & Paso & $R^{2}$ & $r$ & Beta & $t$ & $P$ \\
\hline IAS-Fobia a la enfermedad & $\mathbf{1}$ & 0,19 & 0,18 & 0,21 & 3,65 & $\mathbf{0 , 0 0 0}$ \\
IAS-Preocupaciones corporales & 2 & 0,23 & 0,15 & 0,16 & 3,12 & $\mathbf{0 , 0 0 0}$ \\
EPQR-A-Neuroticismo & 3 & 0,25 & $\mathbf{0 , 1 8}$ & $\mathbf{0 , 1 6}$ & 3,68 & $\mathbf{0 , 0 0 2}$ \\
IAS-Tanatofobia & 3 & 0,26 & $\mathbf{0 , 1 1}$ & $\mathbf{0 , 1 2}$ & $\mathbf{2 , 1 5}$ & $\mathbf{0 , 0 3 3}$ \\
MHLC-Suerte & $\mathbf{5}$ & $\mathbf{0 , 2 7}$ & $\mathbf{0 , 1 1}$ & $\mathbf{0 , 1 0}$ & $\mathbf{2 , 1 3}$ & $\mathbf{0 , 0 3 4}$ \\
\hline
\end{tabular}

Nota: En el modo de regresión no se incluyó la puntuación total de IAS como variable independiente. $R^{2}=$ coeficiente de determinación acumulado. Los valores de los restantes estadísticos ( $r$, beta $t$ y $P$ ) corresponden al último paso. $r=$ coeficiente de correlación parcial. 
Tabla 4. Predicción de las mujeres ansiosas al acudir a la exploración mamográfica de las pruebas complementarias, a partir de las variables sociodemográficas [análisis de regresión logistica simple]

\begin{tabular}{|c|c|c|c|c|c|c|}
\hline Variable independiente & $\chi^{2}$ & $R^{2}$ & $B$ & Wald & OR & IC $95 \%$ \\
\hline Zona geográfica & 5,15 * & 0,02 & & & 100 & \\
\hline Urbana & & & $-0,67$ & 5,54 * & 0,51 & $0,29-0,89$ \\
\hline $\begin{array}{l}\text { Estado civil } \\
\quad \text { Casada/en pareia }\end{array}$ & 8,45 * & 0,03 & & & 1.00 & \\
\hline Soltera & & & $-0,55$ & 2,33 & 0,57 & $0,29-1,17$ \\
\hline Viuda & & & 0,37 & 0,83 & 1,45 & $0,65-3,19$ \\
\hline Separada divorciada & & & $-1,75$ & $2,90^{\mathrm{a}}$ & 0,18 & $0,03-1,30$ \\
\hline Edad & 0,45 & & & & & \\
\hline Ingresos familiares & 5,65 & & & & & \\
\hline Nivel de estudios & 7,65 & & & & & \\
\hline $\begin{array}{l}\text { Número de hijos } \\
\text { Ninguno }\end{array}$ & $9,48^{\mathrm{a}}$ & 0,09 & & & 1,00 & \\
\hline Uno & & & 0,23 & 0,29 & 1,26 & $0,54-2,95$ \\
\hline Dos & & & 0,80 & $5,83 *$ & 2,22 & $1,17-4,24$ \\
\hline Tres & & & 0,50 & 1,84 & 1,65 & $0,80-3,40$ \\
\hline Cuatro & & & 0,92 & $4,46 *$ & 2,53 & $1,07-5,96$ \\
\hline Cinco o más & & & 0,02 & 0,01 & 0,99 & $0,27-3,72$ \\
\hline
\end{tabular}

Nota: Las seis variables categóricas se incluyeron individualmente en los análisis (sólo se indican las categorias en las variables significativas estadísticamente, o con tendencia a la significación). $B=$ coeficiente de regresión logística, $R^{2}=$ coeficiente de determinación, $O R=$ odds ratio, $I C=$ intervalos de confianza del $95 \%$ para los OR. * $p<0,05,{ }^{a} p<0,09$.

urbana reduce en aproximadamente un $50 \%$ el riesgo de estar ansiosa, mientras que el ser viuda lo incrementa en casi un $50 \%$ (comparado con la condición de estar casada o vivir en pareja). Con respecto a la variable número de hijos, aunque los resultados deben tomarse únicamente de forma tentativa ya que el chi-cuadrado no resultó ser significativo, contrastado con no tener hijos se evidencia un incremento del riesgo fundamentalmente en las mujeres con cuatro, dos y tres hijos.
Posteriormente se calculó un análisis de regresión logística múltiple por pasos adelante condicional (criterio de probabilidad para los pasos: de entrada $\leq 0,05$, de salida $\geq$ de 0,10). En el modelo de regresión logística se incluyeron como variables independientes todas las variables psicológicas moduladoras además de las variables sociodemográficas zona geográfica, estado civil y número de hijos. La variable dependiente fue estar (1) o no (0) ansiosa. Los resultados obtenidos indican que únicamente tres variables (fobia a la

Tabla 5. Predicción de las mujeres ansiosas de acudir a la exploración mamográfica de las pruebas complementarias, a partir inicialmente de las variables moduladoras psicológicas y sociodemográficas [análisis de regresión logística múltiple por el método de pasos hacia adelante]

\begin{tabular}{lccccccc}
\hline Variable independiente & Paso & $\chi^{2}$ & $R^{2}$ & $B$ & Wald & OR & IC 95\% \\
\hline IAS-Fobia a la enfermedad & 1 & $44,18^{\star \star *}$ & 0,17 & 0,16 & $9,42^{\star \star}$ & 1,18 & $1,06-1,30$ \\
EPQR-A-Neuroticismo & 2 & $54,59^{\star \star *}$ & 0,20 & $\mathbf{0 , 3 2}$ & $9,911^{\star *}$ & $\mathbf{1 , 3 8}$ & $1,13-1,69$ \\
IAS-Tanatofobia & 3 & $59,83^{\star \star \star}$ & $\mathbf{0 , 2 2}$ & $\mathbf{0 , 1 3}$ & $\mathbf{5 , 1 5 *}$ & $\mathbf{1 , 1 4}$ & $1,02-1,26$ \\
\hline
\end{tabular}

Nota: $\chi^{2}=$ chi-cuadrado del modelo después de cada paso, $R^{2}=$ coeficiente de determinación acumulado, $\mathrm{B}=$ coeficiente de regresión logística, $\mathrm{OR}=$ odds ratio, $\mathrm{IC}=$ intervalos de confianza del $95 \%$ para los OR. Todos los estadísticos, excepto $X^{2}$ y $R^{2}$, corresponden al momento final del análisis (último paso). ${ }^{\star} \mathrm{p}<0,05,{ }^{\star \star} \mathrm{p}<0,01,{ }^{\star \star *} \mathrm{p}<0,001$. 
enfermedad, neuroticismo y tanatofobia) predecían que las mujeres estuvieran o no ansiosas durante el periodo previo a la prueba complementaria (véase la Tabla 5). Por otra parte, los resultados indican que la mayor parte de la varianza era explicada por la variable fobia a la enfermedad (17\%). De acuerdo con los valores de los OR, las tres variables incrementaban ligeramente el riesgo de estar ansiosa (el mayor incremento parecía producirse por el neuroticismo). También se observa que, al igual que ocurría en el análisis de regresión múltiple, las variables sociodemográficas no aportaban ninguna explicación ni valor predictivo más allá del proporcionado por la variables psicológicas. A partir de estas tres variables relevantes, el modelo clasificaba correctamente al $85 \%$ de las mujeres en las categorías de ansiosa y no-ansiosa.
Finalmente se calcularon dos análisis de regresión lineal simple, una con las mujeres del grupo PC y otro para el grupo PR. En los dos análisis, la variable independiente fue la puntuación total del IAS y la variable dependiente la puntuación en ansiedad anticipatoria. Ambos análisis de regresión resultaron ser estadísticamente significativos, si bien tanto el coeficiente beta estandarizado como la varianza explicada fueron mayores en el grupo $P C[\beta=0,48(t=13,3, p=0,000)$, $\left.R^{2}=0,23\right]$ que en el grupo PR $[\beta=0,39(t$ $=10,6, p=0,000), R^{2}=0,16$ ]. En la Figura 1 representamos el diagrama de dispersión y las rectas de regresión con objeto de destacar las diferencias que se dan entre ambos grupos en la relación entre las dos variables (IAS y ansiedad anticipatoria). En la figura se puede observar claramente que las actitudes hipocondríacas (IAS) predicen la ansiedad anticipa-

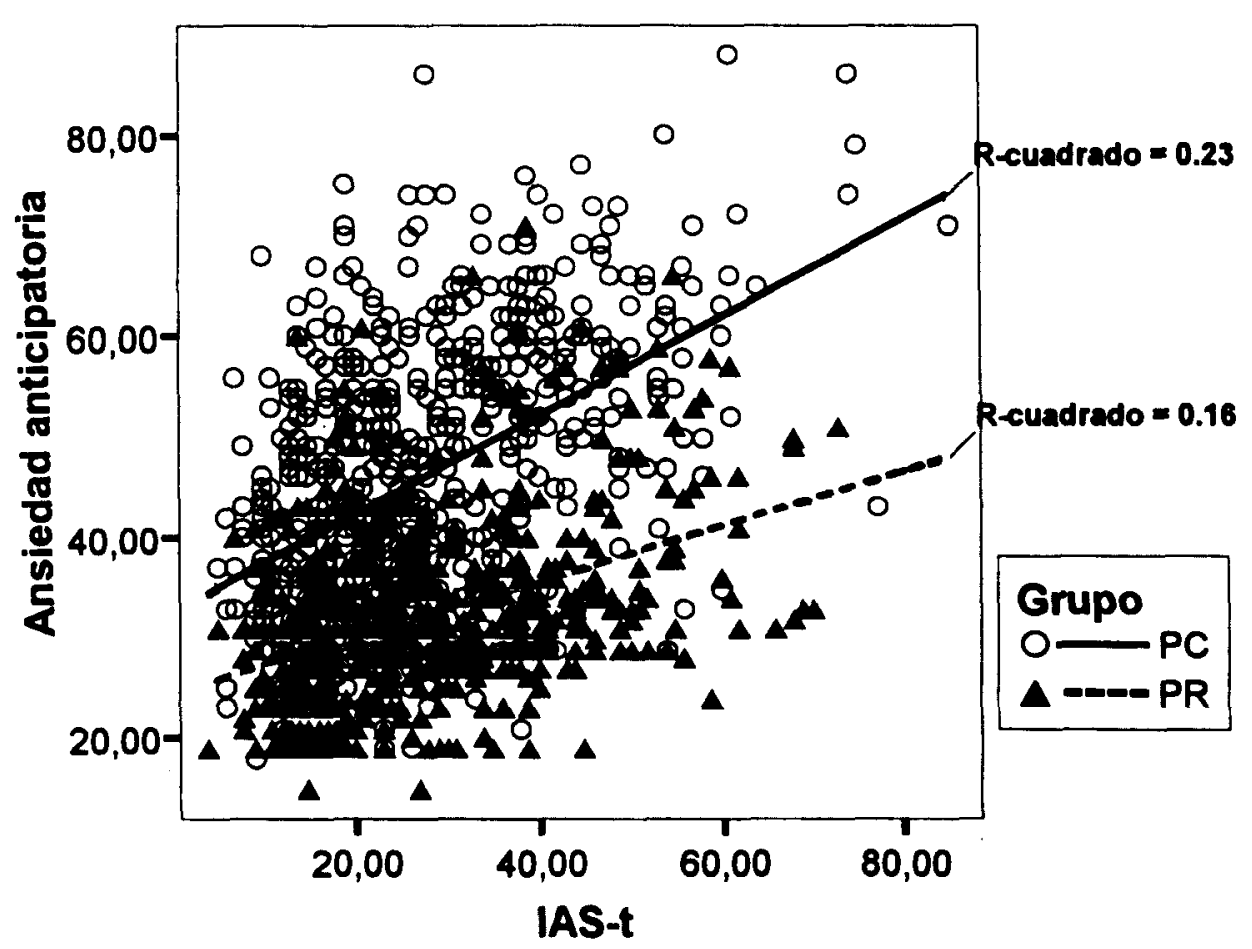

Figura 1. Predicción de la ansiedad anticipatoria a partir de la actitud hacia la enfermedad (IAS; Illness Attitude Scales) en mujeres que participan en pruebas complementarias (grupo PC) o rutinarias (grupo PR) de detección de cáncer de mama. En la figura se indica el diagrama de dispersión y las líneas de regresión de la ansiedad anticipatoria sobre los valores del IAS. 
toria de forma mucho más precisa en las mujeres del grupo PC que en las mujeres del grupo PR.

\section{DISCUSIÓN}

Las tres hipótesis centrales del presente estudio se referían a que: (1) las actitudes hipocondríacas relativas a la dimensión de miedo irreal a la enfermedad deberían ser mejores predictores de la ansiedad anticipatoria ante las pruebas complementarias que las actitudes hipocondríacas relacionadas con la dimensión de convicción de enfermedad; (2) las variables sociodemográficas ingresos familiares, nivel de estudios, edad, número de hijos, vivir en zona urbana, y estar casada o en pareja deberían relacionarse de forma inversa con la ansiedad anticipatoria; y (3) el nivel global de actitud hacia la enfermedad, como principal predictor de la ansiedad anticipatoria asociada al screening de cáncer de mama, debería predecir la ansiedad anticipatoria mejor en las mujeres que acuden a las pruebas complementarias que las que lo hacen a pruebas rutinarias de detección de cáncer de mama.

Los resultados concernientes a nuestros análisis de regresión lineal y logística indican que la variable fobia a la enfermedad es el mejor predictor de la ansiedad anticipatoria en las mujeres que acuden a repetir las pruebas de screening. La variable tanatofobia también ha resultado significativa tanto en el análisis de regresión lineal como en el de regresión logística. La variable preocupaciones corporales sólo ha sido significativa en el análisis de regresión lineal. Ninguna otra variable de actitud hacia la enfermedad proporcionaba algún grado de predicción por encima del aportado por estas variables y el neuroticismo. Puesto que la fobia a la enfermedad y la tanatofobia son los dos principales componentes de la dimensión hipocondríaca de «miedo irreal a la enfermedad» (Belloch y Martínez, 1999; Chorot y Martínez, 1995; Chorot et al., 1997; Kellner, 1986), cabe afirmar que los resultados obtenidos son congruentes con nuestra primera hipótesis. Por otra parte, y como también cabría esperar, los resultados están de acuerdo con la particular relevancia que parecen poseer las variables de actitud hacia la enfermedad para predecir el distress asociado a la participación en las pruebas de detección de cáncer de mama (Lostao, 1994; Sandín et al., 2001c). La diferenciación predictiva asociada a la dimensión de miedo a la enfermedad sugiere la posibilidad de emplear selectivamente esta dimensión en situaciones en que, existiendo cierta amenaza para la salud, esté comprometido el estudio y/o tratamiento de la ansiedad. Por otra parte, estos resultados proporcionan validez discriminante a ambas dimensiones de la hipocondría.

Los análisis en los que están implicados las variables sociodemográficas indican que éstas resultan escasamente relevantes para predecir la ansiedad anticipatoria que sufren las mujeres al acudir a las pruebas. Estas variables no aportan ningún grado de explicación de la varianza, y por tanto ningún valor predictivo, más allá del proporcionado por las variables psicológicas. Estos resultados están en contra de lo que habíamos predicho en nuestra segunda hipótesis, y en general no apoyan mucha de la evidencia que indica que ciertas variables como, por ejemplo, el nivel económico, el apoyo social (p.ej., estar casado o en pareja, número de hijos) y el nivel educativo constituyen importantes factores protectores de la salud cuando el individuo se encuentra ante situaciones estresantes (Anderson y Armstead, 1995; Dohrenwend, 1998; Kaplan, 1995; Lostao et al., 1996; Sandín, 1999). No obstante, merece la pena señalar que, consideradas individualmente, las variables zona 
geográfica y estado civil parecen aportar algún grado de valor predictivo. En este sentido, en línea con lo que habíamos predicho, la pertenencia a una zona rural parece constituir un factor de riesgo para experimentar un nivel elevado de ansiedad. Por otra parte, sin ser concluyentes, nuestros resultados relativos al estado civil indican ligeramente que sólo la condición de ser viuda parece vincularse a un mayor grado de ansiedad anticipatoria.

$\mathrm{La}$ inexistencia de relación entre las dos variables de apoyo social y la ansiedad anticipatoria, a pesar de ir en contra de nuestras predicciones, es congruente con nuestros resultados relativos a algunas de las variables sociodemográficas, tales como el estado civil y el número de hijos. Esto sugiere que el apoyo social es irrelevante en la relación entre el estrés y la salud en el contexto en que se estudia en la presente investigación. Existe evidencia contrastada de que el apoyo social es uno de los factores moduladores (efecto amortiguador) del estrés más importantes (Cohen y Syme, 1985; Krause, 1989; Sandín, 1995, 1999; Taylor y Aspinwall, 1996; Thoits, 1982). Sin embargo, situando el problema en el marco de la presente investigación, aunque el porcentaje de mujeres que sufren ansiedad anticipatoria ante la realización de las pruebas complementarias es importante, los niveles de afectación emocional en general no son elevados (Sandín et al., 2001a, 2001b), lo cual podría explicar la baja significación del apoyo social en este estudio. En un trabajo reciente, Clutton et al. (1999) encontraron, aplicando un modelo de regresión, que la interacción entre el estrés percibido y el apoyo social aportaba cierto grado de predicción del nivel de ansiedad en mujeres que participaban en pruebas complementarias de screening de cáncer de mama. Sin embargo, el apoyo social por sí mismo, tal y como ocurre en el presente estudio, era ineficaz para predecir el nivel de ansiedad que experimentaban las mujeres.

Finalmente, en relación con nuestra tercera hipótesis, hemos obtenido evidencia que apoya la idea de que el nivel global de actitud hacia la enfermedad (evaluado mediante la puntuación total en el cuestionario IAS) predice el nivel de ansiedad anticipatoria de forma más precisa en las mujeres que acuden a las pruebas complementarias que en las que participan en las pruebas rutinarias de detección de cáncer de mama. Este resultado es importante, ya que demuestra que la variable de actitud hacia la enfermedad (o variable de hipocondría) posee cierta especificidad para predecir la ansiedad anticipatoria asociada a situaciones en que pueda estar seriamente comprometida la salud del individuo. Si el IAS ha resultado ser sensible para detectar a las personas hipocondríacas (Kellner, 1986), debe presuponerse que esta variable poseerá mayor capacidad predictiva sobre la ansiedad anticipatoria cuando la amenaza para la salud es más elevada. De hecho, esto es lo que indican nuestros resultados.

Aparte de las posibles implicaciones que puedan suponer los resultados del presente estudio para el conocimiento de los factores psicológicos y sociodemográficos asociados a la ansiedad anticipatoria, en principio se pretende obtener información que pueda ser relevante para mejorar la actuación de los programas de detección de cáncer de mama, programas que, dicho sea de paso, son cada vez más frecuentes y activos en nuestro país, así como también en otros países de Europa y en los Estados Unidos de América. El notable incremento que se ha producido en la participación de las mujeres en estos programas ha supuesto que también se haya incrementado en número de mujeres que, necesariamente, deben repetir las pruebas de mamografía. Los datos que hemos referi- 
do en este y en nuestros anteriores estudios se basan en el PDCM de Navarra. El programa aplica actualmente las segundas pruebas tratando de minimizar el estrés asociado. Por ejemplo, estas pruebas suelen aplicarse en un tiempo máximo de dos días desde que se notifica a la mujer, y el resultado de la segunda mamografía se le facilita casi inmediatamente después de su realización. Tal vez por esto, hemos encontrado que la afectación psicológica (emocional y cognitiva) no es elevada y es transitoria (Sandín et al., 2001b), lo cual proporciona un importante respaldo al funcionamiento y dirección del PDCM. Aun así, hemos visto que un porcentaje significativo de mujeres experimenta niveles de ansiedad entre moderados y elevados (Sandín et al., 2001a). Puesto que la ansiedad anticipatoria experimentada puede reducir significativamente el nivel de bienestar y la calidad de vida de estas mujeres, tal vez cabría mejorarse el programa minimizando el nivel de amenaza para la salud que supone la participación en las pruebas complementarias. Esto podría hacerse incluyendo información específica (p.ej., indicando y justificando que la repetición de la prueba obedece a razones o defectos técnicos, no a sospecha de riesgo). Cualquier otra modificación basada en el manejo de la hipocondría, especialmente de la variante relativa al «miedo a la enfermedad», podría resultar particularmente útil.

\section{REFERENCIAS BIBLIOGRÁFICAS}

American Cancer Society (1998). Cancer facts and figures -1998. Atlanta, GA: Autor.

Anderson, N.B., y Armstead, C.A. (1995). Toward understanding the association of socioeconomic status and health: A new challenge for the biopsychosocial appro- ach. Psychosomatic Medicine, 57, 213225.

Ascunce, N. (1991). Mortalidad por cáncer en España. Movimiento natural de la población española. Prevención secundaria del cáncer. En J. Estapé e I. Burgos (Eds.), Medicina General y Cáncer (pp. 67-75). Barcelona: Doyma.

Ascunce, N., y Del Moral, A. (1993). Compliance in breast cancer screening: The Spanish experience. En A. Gad y M. Rosselli del Turco (Eds.), Breast cancer screening in Europe (pp, 47-53). Berlin: Springer-Verlag.

Belloch, A., y Martínez, M.P. (1999). Trastornos somatoformes: Hipocondría. Madrid: UNED-FUE.

Chamberlain, J., y Palli, D. (1993). Evidence of the effectiveness of breast cancer screening. En A. Gad y M. Rosselli del Turco (Eds.), Breast cancer screening in Europe (pp. 15-23). Berlin: Springer-Verlag.

Chorot, P., y Martínez, P. (1995). Trastornos somatoformes. En A. Belloch, B. Sandín y F. Ramos (Eds.). Manual de psicopatología, Vol. 2 (pp. 225-286). Madrid: McGraw-Hill.

Chorot, P., y Sandín, B. (1989). Escalas de Actitud hacia la Enfermedad de Kellner. Universidad Nacional de Educación a Distancia, Madrid (policopiado).

Chorot, P., Sandín, B., Valiente, R.M., Santed, M.A., y Romero, M. (1997). Actitud hacia la enfermedad, ansiedad y sintomatología somática en pacientes con trastorno de pánico e hipocondría. Revista de Psicopatología y Psicología Clínica, 2, 123-136.

Cioffi, D. (1991). Asymmetry of doubt in medical self-diagnosis: The ambiguity of "uncertain wellness". Journal of Personality and Social Psychology, 61, 969-980.

Clutton, S., Pakenham, K.I., y Buckley, B. (1999). Predictors of emotional well-being following a «false positive» breast cancer screening result. Psychology and Health, 14, 263-275.

Cockburn, J., Staples, M., Hurley, S., y De Luise, T. (1994). Psychological consequences of screening mammography. Journal of Medical Screening, 1, 7-12.

Cohen, S., y Syme, S. (1985). Social support and health. New York: Academic Press.

De Waard, F. (1993). Breast cancer: The scene in Europe. En A. Gad y M. Rosselli del Tur- 
co (Eds.), Breast cancer screening in Europe (pp. 3-5). Berlin: Springer-Verlag.

Dean, C., Roberts, M.M., French, K., y Robinson, S. (1986). Psychiatric morbidity after screening for breast cancer. Journal of Epidemiology Community and Health, 40, 7175.

Dohrenwend, B.P. (1998). Theoretical integration. En B.P. Dohrenwend (Ed.), Adversity, stress and psychopathology (pp. 539555). New York: Oxford University Press.

Ellman, R., Angelin, N., Christians, A., Moss, S., Chamberlain, J., y Maguire, P. (1989). Psychiatric morbidity associated with screening for breast cancer. British Journal of Cancer, 60, 781-784.

Francis, L.J., Brown, B., y Philipchalk, R. (1992). The development of an abbreviated form of the Revised Eysenck Personality Questionnaire (EPQR-A): Its use among students in England, Canada, the U.S.A. and Australia. Personality and Individual Differences, 13, 443-449.

Jiménez, M.P., Sandín, B., Chorot, P., y Santed, M. A. (1994). Perceived social support: Relationship with life events and health. Poster. 23rd International Congress of Applied Psychology. Madrid, 17-22 de julio, p. 421 (Actas de Congreso).

Johstone, K., Brown, J., Gerard, K., O'Hanlon, y Morton, A. (1998). Valuing temporary and chronic health states associated with breast screening. Social Sciences and Medicine, 47, 213-222.

Kaplan, G.A. (1995). Where do shared pathways lead? Some reflections on a research agenda. Psychosomatic Medicine, 57, 208121.

Kellner, R. (1986). Somatization and hypochondriasis. New York: Praeger.

Krause, N. (1989). Issues of measurement and analysis in studies of social support, aging and health. En K.S. Markides y C.L. Cooper (Eds.), Aging, stress and health (pp. 4366). New York: Wiley.

Lerman, C., Trock, B., Rimer, B.K., Jepson, C., Brody, D., y Boyce, A. (1991). Psychological side effects of breast cancer screening. Health Psychology, 10, 259-267.

Lostao, L. (1994). Factores psicosociales asociados a la participación en un programa de prevención de cáncer de mama. Uni- versidad Nacional de Educación a Distancia, Madrid (tesis doctoral no publicada). Lostao, L., Chorot, P., Sandín, B. y Lacabe, F. (1996). Variables psicosociales relacionadas con la participación en un "screening" de cáncer de mama. Arbor $C L I V, 608,33$ 71.

Luengo, S., Lázaro, R., Madero, R., Alvira, F., Fitch, K., Azcona, B., Pérez, J.M., y Caballero, P. (1996). Equity in the access to mammography in Spain. Social Science $y$ Medicine, 43, 1263-1271.

Marteau, T.M. (1994). Psychology and screening: Narrowing the gat between efficacy and effectiveness. British Journal of Clinical Psychology, 33, 1-10.

Martínez, M.P., y Belloch, A. (1993). Relaciones de los miedos y creencias hipocondríacas con ansiedad, depresión y síntomas somáticos. Análisis y Modificación de Conducta, 19, 461-478.

McCaul, K.D., Branstetter, A.D., Schroeder, D.M., y Glasgow, R.E. (1996a). What is the relationship between breast cancer risk and mammography screening? A metaanalytic review. Health Psychology, 15, 423-429.

McCaul, K.D., Reid, P.A., Rathge, R.W., y Martinson, B. (1996b). Does concern about breast cancer inhibit or promote breast cancer screening? Basic and Applied Social Psychology, 18, 183-194.

Rosenberg, M. (1965). Society and the adolescent self-image. Princeton, NJ: Princeton University Press.

Sandín, B. (1995). El estrés. En A. Belloch, B. Sandín y F. Ramos (Eds.), Manual de psicopatología, Vol. 2 (pp. 3-52). Madrid: McGraw-Hill.

Sandín, B. (Ed.) (1999). El estrés psicosocial: Conceptos y consecuencias clínicas. Madrid: Klinik.

Sandín, B., Chorot, P., Valiente, R.M., Lostao, L., y Santed, M.A. (2001a). Ansiedad anticipatoria asociada a las pruebas complementarias de screening de cáncer de mama. Psicothema, 13, 248-254.

Sandín, B., Chorot, P., Valiente, R.M., Lostao, L., y Santed, M.A. (2001b). Adverse psychological effects in women attending $a$ second stage breast cancer screening. Manuscrito enviado para publicación. 
Sandín, B., Chorot, P., Valiente, R.M., Lostao, L., Santed, M.A., y Fernández (2000). Actitudes hipocondríacas en mujeres antes y después de participar en pruebas complementarias de detección de cáncer de mama. Psiquis, 21, 232-240.

Sandín, B., Valiente, R.M., y Chorot, P. (1999). Evaluación del estrés psicosocial. En B. Sandín (Ed.), El estrés psicosocial: Conceptos y consecuencias clínicas (pp. 245316). Madrid: Klinik.

Sandín, B., Valiente, R.M., Chorot, P., y Lostao, L. (1998). Programa de prevención pre$\mathrm{coz}$ de cáncer de mama del Gobierno de Navarra: Afectación psicológica ante las segundas pruebas complementarias de screening. Ponencia presentada al Simposio Estrés y Salud, del II Congreso Iberoamericano de Psicología. Madrid, 13-17 de julio.

Sandín, B., Valiente, R.M., Chorot, P., Lostao, L., y Santed, M.A. (2001c). Predictores de la afectación psicológica asociada a la participación en pruebas complementarias de detección de cáncer de mama. Manuscrito enviado para publicación.

Schmidt, J.G. (1990). The epidemiology of mass breast cancer screening - A plea for a valid measure of benefit. Journal of Clinical Epidemiology, 43, 215-225.

Skrabanek, P. (1985). False premises and false promises of breast cancer screening. Lancet, 316-320.

Spielberger, C.D., Gorsuch, R.R., y Lushene, R.E. (1982). STAI. Cuestionario de Ansiedad Estado/Rasgo. Madrid: TEA.

Sutton, S., Saidi. G., Bickler, G., y Hunter. J. (1995). Does routine screening for breast cancer raise anxiety? Results from a three wave prospective study in England. Journal of Epidemiology and Community Health, 49, 413-418.

Taylor, S.E. (1995). Health psychology. New York: McGraw-Hill.
Taylor, S.E., y Aspinwall, L.G. (1996). Mediating and moderating processes in psychological stress. En H.B. Kaplan (Ed.), Psychosocial stress (pp. 71-110). New York: Academic Press.

Thoits, P. (1982). Conceptual, methodological, and theoretical problems in studying social support as a buffer against life stress. Journal of Health and Social Behavior, 23, 145-159.

Valiente, R.M., Sandín, B., Chorot, P., y Santed, M.A. (1996) Versión española del cuestionario EPQR-Abreviado: Datos preliminares con muestras españolas. Póster. XXXII Congreso de la Sociedad Española de Medicina Psicosomática. Madrid, 21-23 de noviembre.

Valiente, R.M., Sandín, B., Chorot, P., y Santed, M.A. (1997). Cuestionario EPQR-Abreviado (EPQR-A): Validación de la versión española. Póster. I Congreso de la Asociación Española de Psicología Clínica y Psicopatología. Madrid, 3-5 de abril.

Vaux, A., Riedel, S., y Stewart, D. (1987). Modes of social support: The Social Support Behaviors (SS-B) Scale. American Journal of Community Psychology, 15, 209237.

Walker, L.G., Cordiner, C.M., Gilbert, F.J., Needham, G., Deans, H.E., et al. (1994). How distressing is attendance for routine breast screening? Psycho-Oncology, 3, 299304.

Wallston, K.A., Wallston, B.S., y DeVellis, R. (1978). Development of the Multidimensional Health Locus of Control (MHLC) scale. Health Education Monographs, 6, 161-170.

Wardle, J., y Pope, R. (1992). The psychological costs of screening for cancer. Journal of Psychosomatic Research, 36, 609-624.

Warren, R. (1988). The debate over mass mammography in Britain. British Medical Journal, 297, 969-972. 